\title{
Characterization of Non-Manipulable and Pareto Optimal Resource Allocation Strategies for Interference Coupled Wireless Systems
}

\author{
Holger Boche \\ Technical University of Berlin \\ Heinrich Hertz Institute \\ Einsteinufer 25, 10587 Berlin \\ holger.boche@mk.tu-berlin.de
}

\author{
Siddharth Naik \\ Technical University of Berlin \\ Heinrich Hertz Institute \\ Einsteinufer 25, 10587 Berlin \\ naik@ hhi.fraunhofer.de
}

\author{
Tansu Alpcan \\ Technical University of Berlin \\ Deutsche Telekom Laboratories \\ Ernst-Reuter Platz 7, 10587 Berlin \\ alpcan@sec.t-labs.tu-berlin.de
}

\begin{abstract}
This paper investigates the properties of social choice functions that represent resource allocation strategies in interference coupled wireless systems. The allocated resources can be physical layer parameters such as power vectors or antenna weights. Strategy proofness and efficiency of social choice functions are used to capture the respective properties of resource allocation strategy outcomes being non-manipulable and Pareto optimal. In addition, this paper introduces and investigates the concepts of (strict) intuitive fairness and non-participation in interference coupled systems. The analysis indicates certain inherent limitations when designing strategy proof and efficient resource allocation strategies, if the intuitive fairness and nonparticipation are imposed. These restrictions are investigated in an analytical social choice function framework for interference coupled wireless systems. Among other results, it is shown that a strategy proof and efficient resource allocation strategy for interference coupled wireless systems cannot simultaneously satisfy continuity and the frequently encountered property of non-participation.
\end{abstract}

\section{INTRODUCTION}

The operator (and regulator) in wireless systems has a pivotal role to ensure that deployment of technology, services and resources is carried out in a socially optimal manner. Future wireless systems might enable the user equipment with intelligent software allowing the user to choose not to reveal its true value for resources to a controller, e.g. base station, operator. In such wireless systems, the controller serving users has to design and implement resource allocation strategies, extracting the true value of the resources from the users. Such a system model deviates from traditional multiuser system model, where users always reveal there utility for resources honestly.

We utilize the social choice function (SCF) to represent resource allocation strategies in interference coupled wireless systems. The goals of the designed resource allocation strategies can be viewed in terms of social choice, which is simply an aggregation of the reported utilities of the different users towards a single joint decision, namely that of resource allocation.

In the considered wireless system setting, the users can choose their own utility functions. Furthermore, announcing their true utilities to the operator might not be in its best interest of the users. In other words the users can choose to reveal a utility function, which differs from their true utility functions, so as to obtain more utility. Here the theory of mechanism design comes into play. It is interested in designing economic mechanisms, just like computer scientists are interested in designing algorithms. Mechanism design can be thought as reverse game theory and is rather unique within economics in having an engineering perspective. Mechanism design attempts implementing desired social choices in a strategic setting - assuming that the different members of society each act rationally in a game theoretic sense [1].

Non-manipulation of the social choice function outcome, i.e. solution outcome of the resource allocation strategy is captured by the property of strategy proofness. The property of non-manipulation in a multiuser wireless systems implies, that no user has an incentive to misrepresent its utility for resources to the controller, which is allocating the resources. A classical example of a strategy proof SCF is the second price auction (Vickrey Clarke Groves (VCG) auction). Pareto optimality of the solution outcome of the resource allocation strategy is captured by the property of efficiency. This paper studies such and certain other desirable properties of social choice functions representing resource allocation strategies and had the following main contributions:

1) a) A resource allocation strategy for interference coupled wireless systems is non-manipulable emulated by strategy proofness, if and only if the signal-to-interference(-plus-noise) ratio (SINR) function $\gamma_{k}$ for a particular user $k$ is a constant function, independent of the utility of its own utility $u_{k}$.

b) The constant mentioned in (1a) depends on the utility functions of the other users $\boldsymbol{u}_{-k}=$ $\left[u_{1}, \ldots, u_{k-1}, u_{k+1}, \ldots, u_{K}\right]$.

2) We introduce the property of intuitive fairness. Intuitive fairness implies that, if a particular user scales down its demand for utility, then the other users must obtain 
the same or better utility. Strict intuitive fairness is a stricter requirement than intuitive fairness, where users can choose from a family of utility functions (more than scaling their utility functions).

a) The solution outcome of a strategy proof and effcient resource allocation strategy, which satisfies the property of intuitive fairness (see Definition 8 ) is robust to the scaling down the utility of a particular user, when the utility functions of all other users are fixed.

b) A strategy proof and efficient resource allocation strategy, satisfying either intuitive fairness (see Definition 8) or strict intuitive fairness (see Definition 9) results in a solution outcome, which can be changed only if two or more users change their utilities, i.e. the resource allocation strategy is robust to the change in utilities of any singular user.

c) If a strategy proof and efficient resource allocation strategy is not constant with respect to the utility of a particular user $k$, then some other user $j(j \neq k)$ has to pay a price - decrease in its performance, even if this other user $j$ 's utility function is fixed.

3) We introduce the property of non-participation, which says that if a particular user does not demand any utility, then it obtains no resource. Hence, it can be easily seen that the property of non-participation is generally, always satisfied.

A strategy proof and efficient resource allocation strategy for interference coupled wireless systems - cannot simultaneously satisfy continuity and the very natural property of non-participation. Continuity is a desirable property of resource allocation strategies, for designing practical algorithms and for mathematical tractability. Hence, this result proves to be a impossibility result, while designing resource allocation strategies, satisfying strategy proofness, efficiency, continuity and nonparticipation, i.e. a strategy proof, efficient and nonparticipation resource allocation strategy is discontinuous.

We briefly summarize certain previous work done in the framework of wireless or communication systems in relation to strategy proofness - utilizing it as a condition to emulate the property of non-manipulation of a resource allocation strategy. Our reference list is by no means comprehensive and the interested reader is further referred to the references in the mentioned papers. [2] proposes a strategy proof trust management system fitting to wireless ad hoc networks. This system is incentive compatible in which nodes can honestly report trust evidence and truthfully compute and broadcast trust value of themselves and other nodes. [3] develop a general method for turning a primal-dual algorithm into a group strategy proof cost-sharing mechanism. The method is used to design approximately budget balanced cost sharing mechanisms for two NP-complete problems: metric facility location, and single source rent-or-buy network design. Both mechanisms are competitive, group strategyproof and recover a constant fraction of the cost. [4], [5] calls nodes selfish if they are owned by independent users and their only objective is to maximize their individual goals. The paper presents a game theoretic framework for truthful broadcast protocol and strategy proof pricing mechanism. [6] proposes an auction-based admission control and pricing mechanism for priority services, where higher priority services are allocated to the users who are more sensitive to delay, and each user pays a congestion fee for the external effect caused by their participation. The mechanism is proved to be strategy-proof and efficient. [7] addresses the issue of user cooperation in selfish and rational wireless networks using an incentive approach. They present a strategy-proof pricing mechanism for the unicast problem and give a time optimal method to compute the payment in a centralized manner and discuss implementation of the algorithm in the distributed manner. They present a truthful mechanism when a node only colludes with its neighbors. [8], [9] provide a tutorial on mechanism design and attempt to apply it to concepts in engineering. [10], [11] utilize SINR and power auctions to allocate resources in a wireless scenario and present an asynchronous distributed algorithm for updating power levels and prices to characterize convergence using supermodular game theory. [12] have proposed a repeated spectrum sharing game with cheat-proof strategies. They propose specific cooperation rules based on maximum total throughout and proportional fairness criteria.

As can be observed from the above references, they typically design strategy proof resource allocation strategies for particular wireless or communication systems. Our paper characterizes certain inherent boundaries while designing strategy proof and efficient resource allocation strategies, when combined with certain other very desirable and intuitive properties. We investigate these contradictions in the framework of interference coupled wireless systems and an analytical framework of social choice functions, described in Section II below.

\section{Analytical Framework}

In this paper we shall investigate the case of interference coupled wireless systems. Before we begin to describe our system model and present the relevant definitions, we provide certain notational conventions used in the paper in Section II-A below.

\section{A. Preliminaries and Notation}

Matrices and vectors are denoted by bold capital letters and bold lowercase letters, respectively. Let $\boldsymbol{y}$ be a vector, then $y_{l}=[\boldsymbol{y}]_{l}$ is the $l^{\text {th }}$ component. Let $\boldsymbol{y}_{-l}$ denote the vector $\boldsymbol{y}$ without the $l^{\text {th }}$ component. Likewise $G_{m n}=[\boldsymbol{G}]_{m n}$ is a component of the matrix $\boldsymbol{G}$. The notation $\boldsymbol{y} \geq 0$ implies that $y_{l} \geq 0$ for all components $l . \boldsymbol{x}>\boldsymbol{y}$ implies $x_{l}>y_{l}$ for all components $l$. Similar definitions hold for the reverse directions. $\boldsymbol{x} \neq \boldsymbol{y}$ implies that the vector differs in at least one component. The set of non-negative reals is denoted as $\mathbb{R}_{+}$. The set of positive reals is denoted as $\mathbb{R}_{++}$. Let $u, \tilde{u}, f$ 
represent functions. Let $\mathbf{U}$ represent a family of functions. Let $\boldsymbol{u}=\left[u_{1}, \ldots, u_{K}\right]$ represent a $K$-tuple of functions. Let $\mathcal{U}^{K}$ represent a family of $K$-tuple of functions, where $K$ represents the cardinality of the set.

\section{B. Interference Coupled Wireless Systems}

In a wireless system, the users' utilities can strongly depend on the underlying physical layer. An important measure for the link performance is the SINR. Consider $K$ users with transmit powers $\boldsymbol{p}=\left[p_{1}, \ldots, p_{K}\right]^{T}$ and $\mathcal{K}:=\{1, \ldots, K\}$. The noise power at each receiver is $\sigma^{2}$. Hence the SINR at each receiver depends on the extended power vector $\boldsymbol{p}$, where

$$
\underline{\boldsymbol{p}}=\left[\begin{array}{c}
\boldsymbol{p} \\
\sigma^{2}
\end{array}\right]=\left[p_{1}, \ldots, p_{K}, \sigma^{2}\right]^{T} .
$$

The resulting SINR of user $k$ is

$$
\operatorname{SINR}_{k}(\underline{\boldsymbol{p}})=\frac{p_{k}}{\mathcal{I}_{k}(\underline{\boldsymbol{p}})}=\gamma_{k}(\underline{\boldsymbol{p}})
$$

where $\mathcal{I}_{k}$ is the interference (plus noise) as a function of $\boldsymbol{p}$. We describe the framework of these interference functions $\overline{\mathcal{I}}_{k}$ briefly in Section II-C below.

\section{Interference Functions}

In order to model interference coupling, we shall follow the axiomatic approach proposed in [13], [14].

Definition 1. Interference functions: We say that $\mathcal{I}: \mathbb{R}_{+}^{K+1} \mapsto$ $\mathbb{R}_{+}$is an interference function if the following axioms are fulfilled:

$A 1 \quad$ conditional positivity $\mathcal{I}(\underline{p})>0$ if $\underline{p}>\mathbf{0}$

$A 2 \quad$ scale invariance $\mathcal{I}(\alpha \underline{\boldsymbol{p}})=\alpha \mathcal{I}(\underline{\boldsymbol{p}}), \forall \alpha \in \mathbb{R}_{+}$

A3 monotonicity $\mathcal{I}(\underline{\boldsymbol{p}}) \geq \mathcal{I}(\underline{\hat{\boldsymbol{p}}})$ if $\underline{\boldsymbol{p}} \geq \underline{\hat{\boldsymbol{p}}}$

$A 4 \quad$ strict monotonicity $\mathcal{I}(\underline{\boldsymbol{p}})>\mathcal{I}(\underline{\hat{\boldsymbol{p}}})$ if $\boldsymbol{p} \geq \boldsymbol{p}$,

$$
p_{K+1}>\hat{p}_{K+1} \text {. }
$$

Note that we require that $\mathcal{I}(\boldsymbol{p})$ is strictly monotone with respect to the last component $p_{K+1}$. An example is $\mathcal{I}(\boldsymbol{p})=$ $\boldsymbol{v}^{T} \boldsymbol{p}+\sigma^{2}$, where $\boldsymbol{v} \in \mathbb{R}_{+}^{K}$ is a vector of interference coupling coefficients. The axiomatic framework A1-A4 is connected with the framework of standard interference functions [13].

Definition 2. Standard interference functions: A function $Y$ : $\mathbb{R}_{+}^{K} \mapsto \mathbb{R}_{++}$is said to be a standard interference function if the following axioms are fulfilled:

$$
\begin{array}{ll}
Y 1 & \text { positivity } Y(\boldsymbol{p})>0, \text { for all } \boldsymbol{p} \in \mathbb{R}_{+}^{K}, \\
Y 2 & \text { scalability } Y(\alpha \boldsymbol{p})<\alpha Y(\boldsymbol{p}), \text { for all } \alpha>1, \\
Y 3 & \text { monotonicity } Y(\boldsymbol{p}) \geq Y(\hat{\boldsymbol{p}}) \text { if } \boldsymbol{p} \geq \hat{\boldsymbol{p}} .
\end{array}
$$

For any constant noise power $p_{K+1}=\sigma^{2}$ the function $Y(\boldsymbol{p})=\mathcal{I}(\boldsymbol{p})$ is standard. Conversely any standard interference function can be expressed within the framework A1-A4. The details about the relationship between the model A1-A4 and Yates' standard interference functions were discussed in [14] and further investigated in [15]. For the purpose of this paper it is sufficient to be aware that there exists a connection between these two models and the results of this paper are applicable to standard interference functions.

\section{Utility Modeling}

In mathematical economics, the modeling of utilities of the users, is an initial step towards characterizing the preferences of the users and in turn utilizing the framework of mechanism design and implementation theory. We are particularly interested in analyzing the class of utility functions, which is function of SINR, given by (2). The utility functions, which shall be introduced are motivated based on two factors listed below.

- Users in a wireless system coupled by interference and can be competitive in nature.

- Performance indicators in wireless systems are influenced by physical layer parameters.

Definition 3. SINR based utility (SBU) function: For user $k, u_{k}$ is said to be an SBU function, if there exists a strict monotone increasing continuous function $q$ and an interference function $\mathcal{I}_{k}$ such that

$$
u_{k}(\underline{\boldsymbol{p}})=q\left(\frac{p_{k}}{\mathcal{I}_{k}(\underline{\boldsymbol{p}})}\right) .
$$

Let $\boldsymbol{u}=\left[u_{1}, \ldots, u_{K}\right] \in \mathcal{U}^{K}$, where $\mathcal{U}^{K}$ is the family of SBU functions for $K$ users. In our paper, "utility" can represent certain arbitrary performance measures, which depend on the SINR by a strictly monotone and continuous function $\tilde{u}$ defined on $\mathbb{R}_{+}$. The utility of user $k$ is

$$
u_{k}(\underline{\boldsymbol{p}})=\tilde{u}_{k}\left(\gamma_{k}(\underline{\boldsymbol{p}})\right), \quad k \in \mathcal{K} .
$$

An example of the above case if capacity: $\tilde{u}(x)=\log (1+x)$ and effective bandwidth $\tilde{u}(x)=x /(1+x)$. We are usually interested in maximizing the utilities. However, we can think of utilities as loss functions, where we want minimize the loss function. In the following performance indicators, we would like to minimize the loss function, e.g. MMSE: $\tilde{u}(x)=1 /(1+$ $x$ ), BER: $\tilde{u}(x)=Q(\sqrt{x})$ and high-SNR approximation of BER $\tilde{u}(x)=x^{-\alpha}$ with diversity order $\alpha$.

\section{Mechanism Design Framework}

In this section we review certain mechanism design notation introduced in [16] in the context of interference coupled wireless systems. The set of users in the system is defined as $\mathcal{K}:=\{1, \ldots, K\}$. We assume that the number of users $K \geq 2$. Let $\mathcal{R}$ be an arbitrary set of alternatives at the physical layer, where $\mathcal{R}:=\times_{k \in \mathcal{K}} \mathbf{R}_{k}$ and $r_{k} \in \mathbf{R}_{k}$. Resources at the physical layer are power, antenna weights, beamforming vectors etc. A combination of these could also be considered as resources and modeled by our framework.

Example 1. Consider a SIMO virtual uplink scenario with a sum power constraint or a MISO downlink scenario with a sum power constraint $P_{\text {total }}$ and beamforming vectors for the 
users being $\boldsymbol{\omega}_{k}$, for $k \in \mathcal{K}$. The set of resources $\mathcal{R}$ can be represented in this scenario as follows.

$$
\begin{aligned}
\mathcal{R}=\left\{\left(\boldsymbol{p}, \boldsymbol{\omega}_{1}, \ldots, \boldsymbol{\omega}_{K}\right) \mid \boldsymbol{p} \geq \mathbf{0}, \sum_{k \in \mathcal{K}} p_{k} \leq P_{\text {total }},\right. \\
\left.\left\|\boldsymbol{\omega}_{1}\right\|_{2}=\ldots=\left\|\boldsymbol{\omega}_{K}\right\|_{2}=1\right\} .
\end{aligned}
$$

\section{A. Social Choice Functions and Existing Properties of Social Choice Functions}

Each user $k$ has a preference relation defined over the set of alternatives $\mathcal{R}$, which admits a numerical representation $u_{k}: \mathbf{R}_{k} \mapsto \mathbb{R}_{+}$. Different users in a wireless systems could have different preferences as to what they wish the solution outcomes of a resource allocation strategy should be. We utilize a social choice function framework of social choice functions to capture the resource allocation problem in interference coupled wireless systems. We utilize the social choice function to characterize resource allocation strategies. If a particular property (axiom) is satisfied by the social choice function, then the corresponding property is satisfied by the resource allocation strategy, i.e. we utilize certain properties (axioms) to emulate desirable and natural properties of resource allocation strategies. A social choice function (SCF) aggregates the preferences of all the users into a social choice for the entire system, i.e. the solution outcome of a resource allocation strategy [17].

Definition 4. Social choice function: A social choice function (SCF) is a function $f: \mathcal{U}^{K} \mapsto \mathcal{R}$ that associates with every $\boldsymbol{u} \in \mathcal{U}^{K}$ an unique alternative $f(\boldsymbol{u})$ in $\mathcal{R}_{\boldsymbol{x}}$.


Figure 1. Depiction of the set of resources $\mathcal{P}$, mapping of the set of resources to the SINR region and the special case of utilities being scalars (identity functions) mapping to the QoS set in multiuser wireless systems

Example 2. Consider an example, where the resources at the physical layer are only the powers of the users, i.e. $\mathcal{R}=\mathcal{P}$, with $\sum_{k \in \mathcal{K}} p_{k} \leq P_{\text {total }}$ and the utility function is defined by (4). With this scenario, Figure 1 displays the set of resources $\mathcal{P}$, mapping of the set of resources to the SINR region and the special case of utilities being scalars (identity functions) mapping to the QoS set in multiuser wireless systems.

We formalize certain desirable properties of resource allocation strategies by means of an axiomatic framework to capture these properties. Our objective being in exploring the consistencies between the various axioms (properties) of the axiomatic framework which result in permissible resource allocation strategies. The property, that a particular resource allocation strategy is non-manipulable is emulated by the SCF $f$ satisfying the property strategy proofness.

Definition 5. Strategy Proof: A SCF $f$ is said to be strategyproof, if for all users $k \in \mathcal{K}$ and for all utility functions $u_{k}, \hat{u}_{k} \in \mathcal{U}, \forall \hat{\boldsymbol{u}}_{-k} \in \mathcal{U}^{K-1}$, we have that

$$
u_{k}\left(f\left(u_{k}, \hat{\boldsymbol{u}}_{-k}\right)\right) \geq u_{k}\left(f\left(\hat{u}_{k}, \hat{\boldsymbol{u}}_{-k}\right)\right) .
$$

A mechanism is said to be strategy-proof if it reveals the users true utilities.

Definition 6. Efficient: A SCF $f$ is efficient if $\forall \boldsymbol{u} \in \mathcal{U}^{K}$, there is no $\boldsymbol{r} \in \mathcal{R}$ such that $u_{k}(\boldsymbol{r}) \geq u_{k}(f(\boldsymbol{u}))$, for all users $k \in \mathcal{K}$ and $u_{k}(\boldsymbol{r})>u_{k}(f(\boldsymbol{u}))$ for some user $k \in \mathcal{K}$.

Efficiency from the point of view of wireless communication (physical layer perspective) of the resource allocation strategies, implies choosing an operating point on the Pareto boundary of the feasible utility region [18].

Definition 7. Option set: The option set of a user $k \in \mathcal{K}$, given a utility function vector $\boldsymbol{u}_{-k} \in \mathcal{U}^{K}$ is the set

$$
\begin{aligned}
\mathbf{Q}_{k}\left(\boldsymbol{u}_{-k}\right) & = \\
\{\boldsymbol{r} & \left.\in \mathcal{R} \mid \exists u_{k} \in \mathcal{U}, \text { such that } f\left(u_{k}, \boldsymbol{u}_{-k}\right)=\boldsymbol{r}\right\} .
\end{aligned}
$$

where $\boldsymbol{r}$ is a resource vector.

The option set $\mathbf{Q}_{k}$, is the set of resources for all the users, which user $k$ can influence with its utility function, given the utility function vectors $\boldsymbol{u}_{-k} \in \mathcal{U}^{K-1}$. The use of option sets has proved to be a useful technique in analyzing strategy proof SCF [19]. The reader should bear in mind, that option sets are relative to a given function on a given domain, even if this is not explicit in the notation.

\section{B. Introduced Properties of Social Choice Functions}

In this section, we introduce the properties of (strict) intuitive fairness and non-participation and connect them with well established concepts in literature.

Definition 8. Intuitive fairness: A SCF $f$ is said to satisfy the property of intuitive fairness, if for all utility function vectors $\boldsymbol{u} \in \mathcal{U}^{K}$, for all user $k \in \mathcal{K}$ we have that, for arbitrarily chosen $\left(u_{k}, \boldsymbol{u}_{-k}\right)$ and $0<\lambda<1$,

$$
u_{k}\left(f\left(\lambda u_{j}, \boldsymbol{u}_{-j}\right)\right) \geq u_{k}\left(f\left(u_{j}, \boldsymbol{u}_{-j}\right)\right), \quad k \in \mathcal{K}, k \neq j .
$$

A SCF is said to be intuitive fair, if for all users $k \in \mathcal{K}$, we have the case that, if any one of the users linearly scales down its utility, then the other users should either obtain the same or better utility as against the case, when the user had not 
scaled its utility. We now present a stricter form of the above definition, namely that, not only can a user scale its utility function, but it can choose another utility function altogether.

Definition 8 is similar to the axiom of population monotonicity (in the context of cooperative bargaining theory) introduced under a different name in [20]. the axiom of population monotonicity say the following: Suppose a group of users $\mathcal{K}_{1}$ have arrived to play a particular resource allocation game. If the users $\mathcal{K}_{2} \backslash \mathcal{K}_{1}$ (with $\mathcal{K}_{1} \subset \mathcal{K}_{2}$ ) do not show up, let the set of users $\mathcal{K}_{1}$ reach a particular solution outcome to the resource allocation game. If the users $\mathcal{K}_{2} \backslash \mathcal{K}_{1}$ show up afterwards, resource allocation is carried out again and no user in $\mathcal{K}_{1}$ should be better off.

Definition 9. Strict intuitive fairness: A SCF $f$ is said to satisfy the property of strict intuitive fairness, if for all users $k \in \mathcal{K}$, for all utility function vectors $\boldsymbol{u}_{-j} \in \mathcal{U}^{K-1}$, $u_{j}, \hat{u}_{j} \in \mathcal{U}$ and for $0 \leq \hat{u}_{j}(\boldsymbol{r}) \leq u_{j}(\boldsymbol{r})$ for all $\boldsymbol{r} \geq \mathbf{0}$, we have that

$$
u_{k}\left(f\left(\hat{u}_{j}, \boldsymbol{u}_{-j}\right)\right) \geq u_{k}\left(f\left(u_{j}, \boldsymbol{u}_{-j}\right)\right), \quad k \in \mathcal{K} \backslash j .
$$

In the definition of strict intuitive fairness it can be seen, that the utility function $\hat{u}_{j}$ is dominated by the utility function $u_{j}$, for all users $j \in \mathcal{K}$, for all resource vectors $\boldsymbol{r} \in \mathcal{R}$ and utility function vectors $\boldsymbol{u}_{-j} \in \mathcal{U}^{K-1}$.

The properties of intuitive fairness and strict intuitive fairness are somehow connected to the property of maxmin fairness. With any resource allocations strategy providing service quality to the users we can associate a specific notion of fairness. The consideration of fairness notions has been mainly a wired network issue [21], [22]. The most common fairness notion is max-min fairness. It represents an equilibrium associated with an ideally social system characterized by the fact, that no users quality-of-service (QoS) measure can be increased without decreasing an already lower users QoS measure.

Comparing max-min fairness to intuitive fairness, we can see that, if a particular user $k$ reduces its demand for utility, then there are more resources for the remaining users. Hence, another user $j \in \mathcal{K} \backslash k$ could increase its utility without decreasing of an user $m \in \mathcal{K} \backslash\{j, k\}$.

We now discuss another property of resource allocation strategies, namely point-wise continuity. We say that the sequence $\left\{\boldsymbol{u}^{(n)}\right\}_{n \in \mathbb{N}}, \boldsymbol{u}^{(n)} \in \mathcal{U}^{K}$ converges to $\boldsymbol{u} \in \mathcal{U}^{K}$, if for all constants $R_{\text {total }}>0$, we have that

$$
\lim _{n \rightarrow \infty} \max _{\boldsymbol{r} \geq \mathbf{0}, \sum_{k \in \mathcal{K}}^{r_{k} \leq R_{\text {total }}}}\left\|\boldsymbol{u}^{(n)}(\boldsymbol{r})-\boldsymbol{u}(\boldsymbol{r})\right\|_{l^{1}}=0 .
$$

The utility functions $\left\{u_{k}^{(n)}\right\}_{n \in \mathbb{N}}$ are defined by their values, so the utility functions converge if their values converge. This reduces the convergence of real-valued functions to the convergence of real numbers. Such a convergence is called pointwise convergence. Since, we are dealing with utility function vectors (vector of utility functions) as against utility functions. Hence, we use the $l-1$ norm. We would like at this point to remind the reader that, we say that a SCF $f$ is continuous, if for all convergent sequences of utility vectors $\left\{\boldsymbol{u}^{(n)}\right\}_{n \in \mathbb{N}}$ the following expression holds:

$$
\lim _{n \rightarrow \infty}\left\|f\left(\boldsymbol{u}^{(n)}\right)-f(\boldsymbol{u})\right\|_{l^{1}}=0 .
$$

We now explain a very natural property, which is almost always satisfied for all resource allocation strategies occurring in interference coupled wireless systems. The property states that, if a particular user demands no utility, then the resource allocation strategy does not allocate any resource to this user.

Definition 10. Non-participation: A SCF $f$ is said to satisfy the property of non-participation, if for a given user $k \in \mathcal{K}$ and for all utility function vectors $\boldsymbol{u}_{-k} \in \mathcal{U}^{K-1}$, where $\boldsymbol{u}_{-k}$ is not the identically zero $K-1$-tuple, we have that

$$
f_{k}\left(0, \boldsymbol{u}_{-k}\right)=0 .
$$

Equipped with the suitable notations and framework, we present the results of our analysis in Section IV below.

\section{Properties of Resource Allocation Strategies}

\section{A. Non-manipulability and Efficiency}

We begin by presenting a result, which states the following: a SCF $f$ is strategy proof if and only if for all users $k \in \mathcal{K}$, the outcome of the resource allocation for the $k^{\text {th }}$, i.e $\gamma_{k}(\boldsymbol{r})$ is a constant, which is independent of its own utility function $u_{k} \in \mathcal{U}$. However, the constant is dependent on the utility functions $\boldsymbol{u}_{-k} \in \mathcal{U}^{K-1}$ of the other users $j \in \mathcal{K} \backslash k$.

Theorem 1. For all users $k \in \mathcal{K}$, for all utility function vectors $\boldsymbol{u}_{-k} \in \mathcal{U}^{K-1}$, a SCF $f$ is strategy proof, if and only if there exists a constant $c_{k}\left(\boldsymbol{u}_{-k}\right)>0$, where for all resource vectors $\boldsymbol{r} \in \mathbf{Q}_{k}\left(\boldsymbol{u}_{-k}\right), \gamma_{k}(\boldsymbol{r})=c_{k}\left(\boldsymbol{u}_{-k}\right)$, where $\gamma_{k}$ is the SINR function of the $k^{\text {th }}$ user.

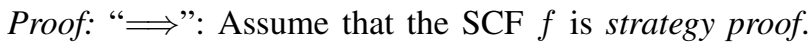
Let there be an arbitrary user $k \in \mathcal{K}$ and an utility function vector $\boldsymbol{u}_{-k} \in \mathcal{U}^{K-1}$ also chosen arbitrarily but fixed. Then, for utility functions $u_{k}, \hat{u}_{k} \in \mathcal{U}$ chosen arbitrarily, we have that

$$
u_{k}\left(f\left(u_{k}, \boldsymbol{u}_{-k}\right)\right) \geq u_{k}\left(f\left(\hat{u}_{k}, \boldsymbol{u}_{-k}\right)\right) .
$$

The above expression follows from strategy proofness. Then we have that,

$$
\gamma_{k}\left(f\left(u_{k}, \boldsymbol{u}_{-k}\right)\right) \geq \gamma_{k}\left(f\left(\hat{u}_{k}, \boldsymbol{u}_{-k}\right)\right) .
$$

However, due to strategy proofness, we also have that

$$
\begin{aligned}
& \hat{u}_{k}\left(f\left(\hat{u}_{k}, \boldsymbol{u}_{-k}\right)\right) \geq \hat{u}_{k}\left(f\left(u_{k}, \boldsymbol{u}_{-k}\right)\right) \\
& \gamma_{k}\left(f\left(\hat{u}_{k}, \boldsymbol{u}_{-k}\right)\right) \geq \gamma_{k}\left(f\left(u_{k}, \boldsymbol{u}_{-k}\right)\right) .
\end{aligned}
$$

Then, we must have that

$$
\begin{aligned}
\gamma_{k}\left(f\left(\hat{u}_{k}, \boldsymbol{u}_{-k}\right)\right) & =\gamma_{k}\left(f\left(u_{k}, \boldsymbol{u}_{-k}\right)\right) \\
& =c_{k}\left(\boldsymbol{u}_{-k}\right) .
\end{aligned}
$$

Since we have chosen the utility vector $\hat{u}_{k} \in \mathcal{U}$ arbitrarily, we have for all resource vectors $\boldsymbol{r} \in \mathbf{Q}_{k}\left(\boldsymbol{u}_{-k}\right)$ that $\gamma_{k}(\boldsymbol{r})=$ $c_{k}\left(\boldsymbol{u}_{-k}\right)$. 
"£": Let us choose an user $k \in \mathcal{K}$ arbitrarily. Let $\boldsymbol{u}_{-k} \in \mathcal{U}^{K-1}$ be an arbitrarily chosen (but fixed) utility function vector. Let $u_{k}, \hat{u}_{k} \in \mathcal{K}$ be chosen arbitrarily. Then, we have that

$$
\begin{aligned}
\gamma_{k}\left(f\left(u_{k}, \boldsymbol{u}_{-k}\right)\right) & =\gamma_{k}\left(f\left(\hat{u}_{k}, \boldsymbol{u}_{-k}\right)\right) \\
u_{k}\left(f\left(u_{k}, \boldsymbol{u}_{-k}\right)\right) & =\tilde{u}_{k}\left(\gamma_{k}\left(f\left(u_{k}, \boldsymbol{u}_{-k}\right)\right)\right) \\
& =\tilde{u}_{k}\left(\gamma_{k}\left(f\left(\hat{u}_{k}, \boldsymbol{u}_{-k}\right)\right)\right) \\
& =u_{k}\left(f\left(\hat{u}_{k}, \boldsymbol{u}_{-k}\right)\right) .
\end{aligned}
$$

Let us choose a user $k \in \mathcal{K}$ arbitrarily, $u_{k}, \hat{u}_{k} \in \mathcal{U}$ and $\boldsymbol{u}_{-k} \in$ $\mathcal{U}^{K-1}$ arbitrarily. Then, it can be easily shown that the SCF $f$ is strategy proof.

We now present a result for 2 users, which shows the restriction of the available SCF $f$, if we want them to satisfy the properties of strategy proofness and efficient, i.e. the solution outcome of the resource allocation strategy is nonmanipulable and is Pareto optimal.

Theorem 2. Let the number of users $K=2$. Then SCF $f$ is efficient and strategy proof, if and only if there exists a resource vector $\boldsymbol{r}^{*} \in \mathcal{R}$ with $\gamma\left(\boldsymbol{r}^{*}\right)$ a Pareto optimal resource allocation and utility function vector $\boldsymbol{u} \in \mathcal{U}^{K}$ with $f(\boldsymbol{u})=\boldsymbol{r}^{*}$.

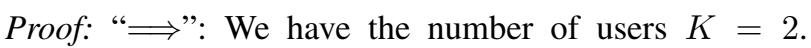
Let SCF $f$ be strategy proof and efficient. For $u_{2} \in \mathcal{U}$ $\left(u_{-1}=u_{2}\right)$ each resource $r \in \mathbf{Q}_{1}\left(u_{-1}\right)$ is on the Pareto boundary $\left(\gamma_{1}(\boldsymbol{r}), \gamma_{2}(\boldsymbol{r})\right)$ of the SINR region. However, we have that $\gamma_{1}(\boldsymbol{r})=c_{1}\left(u_{-1}\right)$ for all resources $r \in \mathbf{Q}_{1}\left(u_{-1}\right)$ and there exists a constant $c_{1}^{(1)}$ with $\gamma_{2}(\boldsymbol{r})=c_{1}^{(1)}\left(u_{-1}\right)$ for all $r \in \mathbf{Q}_{1}\left(u_{-1}\right)$.

Then we have that, for all utility functions $u_{1}, \hat{u}_{1} \in \mathcal{U}$, $f\left(u_{1}, u_{2}\right)=f\left(\hat{u}_{1}, u_{2}\right)$. Let us choose a utility function $\hat{u}_{2} \in \mathcal{U}$ for user 2 arbitrarily. Then, the following expressions hold.

$$
\begin{aligned}
& f\left(u_{1}, u_{2}\right)=f\left(u_{1}, \hat{u}_{2}\right) \\
& f\left(\hat{u}_{1}, u_{2}\right)=f\left(\hat{u}_{1}, \hat{u}_{2}\right) .
\end{aligned}
$$

Therefore, for the utility functions $u_{1}, \hat{u}_{1}, u_{2}, \hat{u}_{2}$ chosen arbitrarily, we have that

$$
\begin{aligned}
f\left(u_{1}, u_{2}\right) & =f\left(\hat{u}_{1}, \hat{u}_{2}\right) \\
& =f\left(\hat{u}_{1}, \hat{u}_{2}\right) .
\end{aligned}
$$

Hence, we have proved our desired result.

"£": Can be easily shown. Hence, we skip the proof.

We now present a result, which displays the existence of a bijective mapping from the domain of the utility function to the range of the utility function, under certain conditions.

Lemma 1. Let power vectors $\underline{\boldsymbol{p}}^{(1)}, \underline{\boldsymbol{p}}^{(2)} \in \mathcal{P}$ be such that $u_{k}\left(\boldsymbol{p}^{(1)}\right)=u_{k}\left(\boldsymbol{p}^{(2)}\right)$, for all users $k \bar{\in} \mathcal{K}$. Then, we have that the powers vectors are equal, i.e $\underline{\boldsymbol{p}}^{(1)}=\underline{\boldsymbol{p}}^{(2)}$.

Proof: We know from the assumptions in the statement of the lemma, that

$$
\begin{aligned}
u_{k}\left(\boldsymbol{p}^{(1)}\right) & =\tilde{u}_{k}\left(\gamma_{k}\left(\boldsymbol{p}^{(2)}\right)\right) \\
& =\tilde{u}_{k}\left(\gamma_{k}\left(\boldsymbol{p}^{(2)}\right)\right) \\
& =u_{k}\left(\boldsymbol{p}^{(2)}\right), \quad k \in \mathcal{K} .
\end{aligned}
$$

Let $\boldsymbol{\Gamma}=\operatorname{diag}\left(\gamma_{1}, \gamma_{2}\right)$. We know that $\gamma_{k}\left(\boldsymbol{p}^{(1)}\right)=\gamma_{k}\left(\boldsymbol{p}^{(2)}\right)$ for $k \in \mathcal{K}$ and $\underline{\boldsymbol{p}}^{(1)}=\boldsymbol{\Gamma} \mathcal{I}\left(\underline{\boldsymbol{p}}^{(1)}\right)$ and $\underline{\boldsymbol{p}}^{(2)}=\boldsymbol{\Gamma} \mathcal{I}\left(\underline{\boldsymbol{p}}^{(2)}\right)$, where $\mathcal{I}$ is a general interference function satisfying the axiom of strict monotonicity in the noise component (axiom $A 4$, refer Section II-B). Then, we have that $\underline{\boldsymbol{p}}^{(1)}=\underline{\boldsymbol{p}}^{(2)}$ from the uniqueness of the fixed point algorithm arguments from Yates [13].

It should be noted here, that we have not exploited any special property of the utility functions, e.g. monotonic increasing. The result only uses the physical layer model of SINR of a user $k$ as $\gamma_{k}=\underline{\boldsymbol{p}} / \mathcal{I}_{k}(\underline{\boldsymbol{p}})$, where $\mathcal{I}_{k}$ is a general interference function for user $k$.

\section{B. Intuitive Fairness and Strict Intuitive Fairness}

Here we present our results in relation to the restrictions obtained, when we try to obtain strategy proof and efficient resource allocation strategies, which satisfy the property of, either

- intuitive fairness or

- strict intuitive fairness.

We now present a result, which states the following: a nonmanipulable, efficient and intuitive fair the solution outcome of a resource allocation strategy is independent of the downwards scaling of the utility function $u_{k} \in \mathcal{U}$ of a particular user $k \in \mathcal{K}$, when the utility functions $\boldsymbol{u}_{-k} \in \mathcal{U}^{K-1}$ of the all the other users $j \in \mathcal{K} \backslash k$ are fixed, i.e. the resource allocation strategy is robust to downwards scaling of the utility function of a particular user, when the utility functions of all the other users are fixed.

Theorem 3. Let a SCF $f$ be strategy proof and efficient. Then, the SCF $f$ fulfills the property of intuitive fair, if and only if for all users $k \in \mathcal{K}$, for all utility functions $\boldsymbol{u} \in \mathcal{U}^{K}$ and for $0<\lambda \leq 1$, we have that

$$
u_{k}\left(f\left(\lambda u_{k}, \boldsymbol{u}_{-k}\right)\right)=u_{k}\left(f\left(u_{k}, \boldsymbol{u}_{-k}\right)\right), \quad 1 \leq k \leq \mathcal{K},
$$

i.e. for $0<\lambda \leq 1$ we have that $f\left(\lambda u_{k}, \boldsymbol{u}_{-k}\right)=f\left(u_{k}, \boldsymbol{u}_{-k}\right)$.

Proof: Let SCF $f$ be strategy proof, efficient and not a constant function. Let us assume that SCF $f$ is $x$ fair. Then, we have that for all users $k \in \mathcal{K}$, for all utility function vectors $\boldsymbol{u} \in \mathcal{U}^{K}$ for $\boldsymbol{u}(\lambda)=\left(\lambda u_{k}, \boldsymbol{u}_{-k}\right), 0<\lambda \leq 1$ and for all users $j \in \mathcal{K} \backslash k$, we have that

$$
u_{k}(f(\boldsymbol{u}(\lambda))) \geq u_{k}(f(\boldsymbol{u})) .
$$

Furthermore, we have that $\gamma_{k}(f(\boldsymbol{u}(\lambda))) \geq \gamma_{k}(f(\boldsymbol{u}))$. For user $j$ we have from Theorem 1 , that $\gamma_{k}(f(\boldsymbol{u}(\lambda)))=\gamma_{k}(f(\boldsymbol{u}))$. Then, for $\boldsymbol{r}(\lambda):=f(\boldsymbol{u}(\lambda))$ we have that $u_{k}(\boldsymbol{r}(\lambda)) \geq$ $u_{k}(f(\boldsymbol{u}))$ for $k \in \mathcal{K}$. Since, SCF $f$ is efficient, we must have that $u_{k}(\boldsymbol{r}(\lambda))=u_{k}(f(\boldsymbol{u}))$ for $k \in \mathcal{K}$.

We know present a Corollary to Theorem 3, which states the following: Let a solution outcome to a resource allocation strategy be non-manipulable and efficient. If the resource allocation strategy is not robust to downward scaling of the utility function of a particular user $k \in \mathcal{K}$, then at least one another user $j \in \mathcal{K} \backslash k$ pays a price with a decrease in its performance, even if the utility functions $\boldsymbol{u}_{-k}$ are fixed. 
Corollary 1. Let $S C F f$ be strategy proof and efficient. For arbitrarily chosen user $k \in \mathcal{K}, u_{k} \in \mathcal{U}, \boldsymbol{u}_{-k} \in \mathcal{U}^{K-1}$ and $\hat{\lambda} \in(0,1)$, let

$$
f\left(\hat{\lambda} u_{k}, \boldsymbol{u}_{-k}\right) \neq f\left(u_{k}, \boldsymbol{u}_{-k}\right) .
$$

Then, there exists at least one user $j \in \mathcal{K} \backslash k$ such that

$$
u_{j}\left(f\left(\hat{\lambda} u_{k}, \boldsymbol{u}_{-k}\right)\right)<u_{j}\left(f\left(u_{k}, \boldsymbol{u}_{-k}\right)\right) .
$$

Proof: Let the assumptions of the corollary be true. Let us assume that, for all $k \in \mathcal{K}$, for all utility vectors $u_{k} \in \mathcal{U}$, for all utility function vectors $\boldsymbol{u}_{-k} \in \mathcal{U}^{K-1}$ and for all $j \in \mathcal{K} \backslash k$ with $\lambda \in(0,1)$ we have that

$$
u_{j}\left(f\left(\lambda u_{k}, \boldsymbol{u}_{-k}\right)\right) \geq u_{j}\left(f\left(u_{k}, \boldsymbol{u}_{-k}\right)\right) .
$$

Since, SCF $f$ satisfies the axioms of strategy proofness and efficiency, we have that

$$
u_{j}\left(f\left(\lambda u_{k}, \boldsymbol{u}_{-k}\right)\right)=u_{j}\left(f\left(u_{k}, \boldsymbol{u}_{-k}\right)\right), \quad j \in \mathcal{K} \backslash k .
$$

From Theorem 1 we have for an arbitrarily chosen user $k$, that

$$
u_{k}\left(f\left(\lambda u_{k}, \boldsymbol{u}_{-k}\right)\right)=u_{k}\left(f\left(u_{k}, \boldsymbol{u}_{-k}\right)\right) .
$$

Furthermore, we have that

$$
f\left(\lambda u_{k}, \boldsymbol{u}_{-k}\right)=f\left(u_{k}, \boldsymbol{u}_{-k}\right)
$$

for $0<\lambda<1$.

We now present certain results, in relation to the stronger property of strict intuitive fairness.

Theorem 4. Let a SCF $f$ be strategy proof and efficient. $S C F f$ fulfills the property of strict intuitive fairness, if for an arbitrary user $k \in \mathcal{K}$, for all $j \in \mathcal{K} \backslash k$ with utility function vector $\boldsymbol{u}_{-k} \in \mathcal{U}^{K-1}$, there exists a constant $d_{k}\left(\boldsymbol{u}_{-k}, j\right)$ such that for all resources $\boldsymbol{r} \in \mathbf{Q}_{k}\left(\boldsymbol{u}_{-k}\right)$ we have that

$$
u_{k}(\boldsymbol{r})=d_{k}\left(\boldsymbol{u}_{-k}, j\right) \text {. }
$$

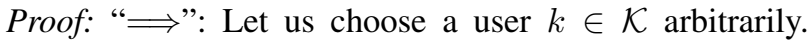
We shall take the perspective of user $k$ without any loss of generality. Let us arbitrarily choose a utility function vector $\boldsymbol{u}_{-k} \in \mathcal{U}^{K-1}$. We have to show that for utility functions $u_{k}, \hat{u}_{k} \in \mathcal{U}$, the following expression (10) holds.

$$
u_{k}\left(f\left(u_{k}, \boldsymbol{u}_{-k}\right)\right)=u_{k}\left(f\left(\hat{u}_{k}, \boldsymbol{u}_{-k}\right)\right), \quad k \in \mathcal{K}
$$

Let us assume that there exists a user $k_{0}$, where $k_{0} \in \mathcal{K} \backslash k$, such that

$$
u_{k_{0}}\left(f\left(u_{k}, \boldsymbol{u}_{-k}\right)\right) \neq u_{k_{0}}\left(f\left(\hat{u}_{k}, \boldsymbol{u}_{-k}\right)\right) .
$$

We define $u_{k}^{*}(\boldsymbol{r})$ as follows:

$$
u_{k}^{*}(\boldsymbol{r})=\max \left(u_{k}(\boldsymbol{r}), \hat{u}_{k}(\boldsymbol{r})\right) .
$$

The utility function $u_{k}^{*}$ is strictly monotonic increasing and continuous. For all resource vectors $r \in \mathcal{R}$, we have that $u_{k}^{*}(\boldsymbol{r}) \geq u_{k}(\boldsymbol{r})$ and $u_{k}^{*}(\boldsymbol{r}) \geq \hat{u}_{k}(\boldsymbol{r})$. Therefore, from the property of intuitive fairness for all users $j \in \mathcal{K} \backslash k$, we have that

$$
\begin{aligned}
& u_{j}\left(f\left(u_{k}^{*}, \boldsymbol{u}_{-k}\right)\right) \leq u_{j}\left(f\left(u_{k}, \boldsymbol{u}_{-k}\right)\right), \quad j \in \mathcal{K} \backslash k, \\
& \gamma_{j}\left(f\left(u_{k}^{*}, \boldsymbol{u}_{-k}\right)\right) \leq \gamma_{j}\left(f\left(u_{k}, \boldsymbol{u}_{-k}\right)\right), \quad j \in \mathcal{K} \backslash k .
\end{aligned}
$$

From Theorem 1 we have that

$$
\gamma_{k}\left(f\left(u_{k}^{*}, \boldsymbol{u}_{-k}\right)\right) \leq \gamma_{k}\left(f\left(u_{k}, \boldsymbol{u}_{-k}\right)\right) .
$$

Since, SCF $f$ is efficient, we must have that

$$
\gamma_{k}\left(f\left(u_{k}^{*}, \boldsymbol{u}_{-k}\right)\right)=\gamma_{k}\left(f\left(u_{k}, \boldsymbol{u}_{-k}\right)\right), \quad \forall k \in \mathcal{K} .
$$

Therefore, from Theorem 1, we have that

$$
f\left(u_{k}^{*}, \boldsymbol{u}_{-k}\right)=f\left(u_{k}, \boldsymbol{u}_{-k}\right) .
$$

We can have a same expression also for $\left(u_{k}^{*}, \boldsymbol{u}_{-k}\right)$ and $\left(\hat{u}_{k}, \boldsymbol{u}_{-k}\right)$. Then, for arbitrary utility functions $u_{k}, \hat{u}_{k} \in \mathcal{U}$ we have that

$$
f\left(u_{k}, \boldsymbol{u}_{-k}\right)=f\left(\hat{u}_{k}, \boldsymbol{u}_{-k}\right) .
$$

We have proved the desired result.

"£": It can be easily shown that a strategy proof and efficient SCF $f$ satisfies the property of strict intuitive fairness.

From the above proof, we can obtain the following additional result, which states that, if a resource allocation strategy satisfies the properties of strategy proofness, efficiency and strict intuitive fairness, then changing the preference of a single user is not sufficient to change the resource allocation, i.e. to affect a change in the resource allocation at least two users must change their preferences or utility functions for the desired resources.

Theorem 5. Let a SCF $f$ be strategy proof and efficient. The $S C F \quad f$ fulfills the property of strict intuitive fairness, if for all user $k \in \mathcal{K}$, for all utility function vectors $\boldsymbol{u}_{-k} \in \mathcal{U}^{K-1}$, we have that cardinality of the option set $\mathbf{Q}_{k}\left(u_{k}\right)=1$. Therefore, for any utility functions $u_{k}, \hat{u}_{k} \in \mathcal{U}$ we have that

$$
f\left(u_{k}, \boldsymbol{u}_{-k}\right)=f\left(\hat{u}_{k}, \boldsymbol{u}_{-k}\right) .
$$

Proof: The proof is a contained in the proof of Theorem 4.

We have stated that, for all utility function vectors $\boldsymbol{u}_{-k} \in$ $\mathcal{U}^{k-1}$, for all utility functions $u_{k} \neq 0$ and for an arbitrarily chosen user $j \in \mathcal{K} \backslash k$, we have that

$$
\begin{aligned}
\inf _{\boldsymbol{r} \in \mathbf{Q}_{k}\left(\boldsymbol{u}_{-k}\right)} r_{k} & =\inf _{u_{k} \neq 0} f_{k}\left(\left(u_{k}, \boldsymbol{u}_{-k}\right)\right) \\
& >0 .
\end{aligned}
$$

Theorem 5 has certain connection to the axiom - non-dummy introduced in [23]. A mechanism $f$ is non-dummy, if $\forall k \in \mathcal{K}$, $\exists \boldsymbol{u} \in \mathcal{U}^{K}$ and $\hat{u}_{k} \in \mathcal{U}$, such that

$$
f(\boldsymbol{u}) \neq f\left(\hat{u}_{k}, \boldsymbol{u}_{-k}\right) .
$$

The non-dummy axiom states that, each user can change the outcome of the mechanism by changing its utility function. It guarantees every user the minimum right to affect the social decision. Then, we can say that a strategy proof, efficient and strict intuitive fair resource allocation strategy does not satisfy the axiom non-dummy. 


\section{Non-participation}

In this section we present a result (Theorem 6), which states that if the solution outcome of the resource allocation strategy is non-manipulable, Pareto optimal and satisfies the natural property of non-participation, then the resource allocation strategy has to be discontinuous. This has serious implications on the practical design of algorithms implementing resource allocation strategies. Furthermore, for theoretical investigation and analysis - continuity is a very desirable property for resource allocation strategies. In certain classes of widely used games, the Nash equilibrium is a continuous function of the game parameters, which follows from the implicit function theorem [24], [25].

While investigating the property of non-participation, we impose a further requirement on the range of the SCF $f$ as follows.

Requirement 1. Let $f\left(\mathcal{U}^{K}\right) \cap \mathbb{R}_{++}^{K} \neq \emptyset$, i.e. there exists a $K-$ tuple of utility functions $\hat{\boldsymbol{u}}=\left[\hat{u}_{1}, \ldots, \hat{u}_{K}\right]$ such that $f_{k}(\hat{\boldsymbol{u}})>$ 0 for all users $k \in \mathcal{K}$.

If we want to investigate the property of non-participation, it is natural to expect that there exists at least one $K$-tuple of utility functions such that all users all users obtain a non-zero resource. Then, according to the property of non-participation we let one arbitrary user demand zero utility and see the impact on the resource allocation for the system.

Example 3. Consider the case of a resource region corresponding to the 2 user case with a total power constraint. Let $r_{1}^{\max }$ and $r_{2}^{\max }$ be the maximum available resources to user 1 and user 2, respectively, while fulfilling the total power constraint. Let the two points $\boldsymbol{r}^{(1)}, \boldsymbol{r}^{(2)} \in \mathcal{R}$ be the range of the SCF $f$, where $\boldsymbol{r}^{(1)}=\left[r_{1}^{\max }, 0\right]$ and $\boldsymbol{r}^{(2)}=\left[0, r_{2}^{\max }\right]$. Let an SCF $f$ satisfy the property of efficiency. It can be clearly seen, that the SCF $f$ is discontinuous and does not satisfy Requirement 1.

Theorem 6. Let a SCF $f$ be strategy proof and efficient. Let requirement 1 be satisfied. Then, the SCF $f$ cannot simultaneously be continuous and satisfy the property of nonparticipation.

Proof: Let a SCF $f$ be strategy proof and efficient. For the sake of obtaining a contradiction, let us assume that the SCF $f$ is continuous and satisfies the property of non-participation. Let us choose a user $k \in \mathcal{K}$ arbitrarily and take the perspective of this user $k$, without any loss in generality. Let us choose a utility function vector $\boldsymbol{u}_{-k} \in \mathcal{U}^{K-1}$ such that $f_{k}\left(u_{k}, \boldsymbol{u}_{-k}\right)>$ 0 .

For all power vectors $\underline{\boldsymbol{p}} \in \mathbf{Q}_{k}\left(\boldsymbol{u}_{-k}\right)$ we have that

$$
\gamma_{k}(\underline{\boldsymbol{p}})=c_{k}\left(\boldsymbol{u}_{-k}\right)>0 .
$$

Therefore, we have that

$$
\frac{p_{k}}{\mathcal{I}_{k}(\underline{\boldsymbol{p}})}=c_{k}\left(\boldsymbol{u}_{-k}\right),
$$

for all power vectors $\boldsymbol{p} \in \mathbf{Q}_{k}\left(\boldsymbol{u}_{-k}\right)$. Exploiting the fact, that $\mathcal{I}_{k}$ is an interference function, we have that

$$
\begin{aligned}
\mathcal{I}_{k}(\underline{\boldsymbol{p}}) & =\mathcal{I}_{k}\left(\left(\boldsymbol{p}, \sigma_{k}^{2}\right)\right) \\
& \geq \mathcal{I}_{k}\left(\left(\mathbf{0}, \sigma_{k}^{2}\right)\right) \\
& =\sigma_{k}^{2} \mathcal{I}_{k}((\mathbf{0}, 1)) \\
& =\sigma_{k}^{2} \mu_{k}, \quad 0<\mu_{k}=\mathcal{I}_{k}((\mathbf{0}, 1)) \\
& >0 .
\end{aligned}
$$

For all power vectors $\underline{\boldsymbol{p}} \in \mathbf{Q}_{k}\left(\boldsymbol{u}_{-k}\right)$ we have that

$$
\begin{aligned}
c_{k}\left(\boldsymbol{u}_{-k}\right) & =\frac{p_{k}}{\mathcal{I}_{k}(\underline{\boldsymbol{p}})} \\
& \leq \frac{p_{k}}{\sigma_{k}^{2} \mu_{k}},
\end{aligned}
$$

where $\mu_{k}=\mathcal{I}_{k}((\mathbf{0}, 1))$. Therefore, we have that the power vector $p_{k} \geq c_{k}\left(\boldsymbol{u}_{-k}\right) \sigma_{k}^{2} \lambda_{k}$, where $\lambda \in(0,1)$. Let $\boldsymbol{u}(\lambda)(\underline{p})=$ $\left(\lambda u_{k}, \boldsymbol{u}_{-k}\right)(\underline{\boldsymbol{p}})$ for all $P_{\text {total }}>0$. Then, we have that

$$
\begin{array}{r}
\lim _{\lambda \rightarrow 0}\left(\max _{\boldsymbol{p} \geq \mathbf{0}, \sum_{k \in \mathcal{K}} p_{k} \leq P_{\text {total }}}\left\|\boldsymbol{u}(\lambda)(\underline{\boldsymbol{p}})-\left(0, \boldsymbol{u}_{-k}\right)(\underline{\boldsymbol{p}})\right\|_{l^{1}}\right)= \\
\lim _{\lambda \rightarrow 0}\left(\lambda \max _{\boldsymbol{p} \geq \mathbf{0}, \sum_{k \in \mathcal{K}} p_{k} \leq P_{\text {total }}}\left|u_{k}(\underline{\boldsymbol{p}})\right|\right)=0 .
\end{array}
$$

Then, we have that

$$
\begin{aligned}
\lim _{\lambda \rightarrow 0} f_{k}(\boldsymbol{u}(\lambda)) & =f_{k}\left(\left(0, \boldsymbol{u}_{-k}\right)\right) \\
& =0 .
\end{aligned}
$$

Equation (13), follows from the property of non participation (Definition 10), which we have assumed that our SCF $f$ satisfies (for the sake of obtaining a contradiction). However, we have that

$$
\begin{aligned}
f_{k}(\boldsymbol{u}(\lambda)) & \geq c_{k}\left(\boldsymbol{u}_{-k}\right) \sigma_{k}^{2} \mu_{k} \\
& >0 .
\end{aligned}
$$

Furthermore, we have that $\inf _{0<\lambda<1} f_{k}(\boldsymbol{u}(\lambda))>0$, which is in contradiction with (13). Hence, we have our desired contradiction, which proves the result.

\section{CONClusion}

This paper investigates certain desirable and natural properties of social choice functions representing resource allocation strategies in interference coupled wireless systems. The properties of non-manipulation and Pareto optimality of the solution outcome of resource allocation strategies is captured by the properties of strategy proofness and efficiency of the social choice function, respectively.

We introduce three desirable and natural properties of resource allocation strategies, namely (strict) intuitive fairness and non-participation. We prove that there are certain inconsistencies, among the properties of strategy proofness, efficiency, (strict) intuitive fairness, non-participation and continuity. This paper has proved that these inconsistencies result in certain limitations while having algorithmic implementations and certain analytical investigations of resource 
allocation strategies. Hence, it can be observed that nonmanipulation and Pareto optimality of the solution outcome of resource allocation strategies are stringent requirements and along with certain other desirable properties is not always implementable.

\section{ACKNOWLEDGMENTS}

Holger Boche and Tansu Alpcan were supported in part by the project Spectrum Allocation Games (SAGa) in cooperation with Deutsche Telekom Laboratories.

Siddharth Naik was supported by the DFG project BO $1734 / 17-1$.

\section{REFERENCES}

[1] N. Nissan, T. Roughgarden, and E. Tardos, Algorithmic Game Theory. Cambridge University Press, 2007.

[2] S. Huiping and S. Junde, "Strategyproof Trust Management in Wireless Ad hoc Network," in Electrical and Computer Engineering, 2004. Canadian Conference on, vol. 3, May 2004, pp. 1593-1596.

[3] M. Pal and E. Tardos, "Group Strategy Proof Mechanisms via PrimalDual Algorithms," in Foundations of Computer Science, 2003. Proceedings. 44th Annual IEEE Symposium on, October, pp. 584-593.

[4] N. R. Suri, "Design of Incentive Compatible Protocols for Wireless Networks: A Game Theoretic Approach," in IEEE International Confernce on Computer Communications (INFOCOM), April 2006, pp. 1-2.

[5] N. R. Suri, Y. Narahari, and D. Manjunath, "An Efficient Pricing Based Protocol for Broadcasting in Wireless Ad hoc Networks," in Communication System Software and Middleware, 2006. Comsware 2006. First International Conference on, January 2006, pp. 1-7.

[6] Z. Guanxiang, L. Yan, Y. Zongkai, and C. Wenqing, "Auction-Based Admission Control and Pricing for Priority Services," in Local Computer Networks, 2004. 29th Annual IEEE International Conference on, November 2004, pp. 398-399.

[7] W. Z. Wang and X. Y. Li, "Truthful Low-Cost Unicast in Selfish Wireless Networks," in Parallel and Distributed Processing Symposium, 2004. Proceedings. 18th International, April 2004, p. 219.

[8] D. Garg, Y. Narahari, and S. Gujar, "Foundations of Mechanism Design: A Tutorial Part 1 - Key Concepts and Classical Results," Sadhana, vol. 33, no. 3, pp. 83-130, April 2008.

[9] — - "Foundations of Mechanism Design: A Tutorial Part 2 - Advanced Concepts and Results," Sadhana, vol. 33, no. 2, pp. 131-174, April 2008

[10] J. Huang, R. Berry, and M. Honig, "Distributed Interference Compensation for Wireless Networks," IEEE Journal on Selected Areas in Communications, vol. 24, no. 5, pp. 1074-1084, May 2006.

[11] _ " "Auction-based Spectrum Sharing," ACM Mobile Networks and Applications Journal, vol. 24, no. 5, pp. 405-418, June 2006.

[12] Y. Wu, B. Wang, K. J. R. Liu, and T. C. Clancy, "Repeated Open Spectrum Sharing Game with Cheat-Proof Strategies," IEEE Transactions on Wireless Communications, vol. 8, no. 4, pp. 1922-1933, April 2009.

[13] R. D. Yates, "A Framework for Uplink Power Control in Cellular Radio Systems," IEEE Journal on Selected Areas in Communication, 1995.

[14] M. Schubert and H. Boche, "QoS-Based Resource Allocation and Transceiver Optimization." Foundations and Trends in Communications and Information Theory, 2005/2006, vol. 2, no. 6, pp. 383-529.

[15] H. Boche and M. Schubert, "Unified Framework for Interference Modeling of Multi-User Wireless Networks," in IEEE International Conference on Communications (ICC), Dresden, Germany, June 2009.

[16] T. Saijo, T. Sjoestroem, and T. Yamato, "Secure Implementation," Theoretical Economics, vol. 2, pp. 203-229, 2007.

[17] E. Maskin, How to Implement Social Goals, Berlin - Brandenburgische Akademie der Wissenschaft, Berichte und Abhandlungen. Akademisches Verlag GmBH, 2009, pp. 9-27, this article is a revised version of Eric Maskin's Nobel Prize Lecture delivered on December 8, 2007 in Stockholm.

[18] — , "Nash Equilibrium and Welfare Optimality," Review of Economic Studies, vol. 66, pp. 23-38, 1999.

[19] S. Babera and B. Peleg, "Strategy-Proof Voting Schemes with Continuous Preferences," Social Choice and Welfare, vol. 7, pp. 31-38, 1990

[20] W. Thomson, "The Fair Division of a Fixed Supply Among a Growing Population," Mathematics of Operation Research, vol. 8, pp. 319-326, 1983.
[21] J. Mo and J. Walrand, "Fair End-to-End Window-Based Congestion Control," IEEE/ACM Transactions on Networking, vol. 5, no. 8, pp. 556-567, October 2000.

[22] L. Massoulie and J. Roberts, "Bandwidth Sharing: Objectives and Algorithms," IEEE/ACM Transactions on Networking, vol. 3, no. 10, pp. 320-328, June 2002.

[23] M. Kato and S. Ohseto, "Non-Dummy Agents in Pure Exchange Economies," The Japanese Economic Review, vol. 55, no. 2, pp. 212 220, June 2004

[24] L. Kudryavtsev, Encyclopaedia of Mathematics, M. Hazewinkel, Ed. Springer-Verlag, 2002.

[25] T. Alpcan, L. Pavel, and N. Stefanovic, "A Control Theoretic Approach to Noncooperative Game Design," in IEEE Conference on Decision and Control, Shanghai, China, December 2009. 